\title{
Calcium Regulatory Protein, Regucalcin, may be a Key Molecule in Brain Disease
}

\author{
Masayoshi Yamaguchi* \\ Department of Hematology and Medical Oncology, Emory University School of Medicine,, Atlanta, USA
}

Regucalcin was discovered in 1978 as a novel calcium-binding protein [1-4]. Regucalcin (RGN) and its gene ( $r g n)$ are identified in over 15 species of vertebrate and invertebrates consisting of regucalcin family [5]. Regucalcin plays a multifunctional role in cell regulation; maintaining of intracellular calcium $\left(\mathrm{Ca}^{2+}\right)$ homeostasis, suppressions of cell signaling, protein synthesis, nuclear deoxyribonucleic acid and ribonucleic acid synthesis and cell proliferation and apoptosis, and regulation of gene expression in various tissue and cell types [6,7]. Regucalcin has been proposed to play a pivotal role as a suppressor protein in signaling systems in maintaining of cell homeostasis [7]. The regucalcin gene is located on the $\mathrm{X}$ chromosome [8,9]. Interestingly, the map location for a growing number of diseases with a genetic basis is encompassed in human X chromosome, and these are known to include syndromic and non-syndromic forms of X-linked mental retardation and X-linked neuromuscler diseases [9]. Regucalcin is expressed in rat brain neuron, and it is decreased in the cerebral cortex and hippocampus of brain with aging $[10,11]$. There is growing evidence that regucalcin plays a pivotal role in brain neuronal cell regulation.

Neuronal $\mathrm{Ca}^{2+}$ homeostasis and $\mathrm{Ca}^{2+}$ signaling regulate multiple neuronal functions, including synaptic transmission, plasticity and cell survival [12]. $\mathrm{Ca}^{2+}$-dependent biochemical processes have been implicated in mechanisms of neuronal plasticity like long-term potentiation, which is likely to play an important role in learning and memory [13]. These multiple functions of $\mathrm{Ca}^{2+}$ require fine regulation of its free intracellular concentration. Intracellular $\mathrm{Ca}^{2+}$ concentration in the neuronal cells of brain is regulated by various buffering and transport systems such as the membrane $\mathrm{Na}^{+}-\mathrm{Ca}^{2+}$ exchanges, the membranous $\mathrm{Ca}^{2+}$-ATPase, $\mathrm{Ca}^{2+}$-binding proteins, and intracellular $\mathrm{Ca}^{2+}$ uptake systems $[14,15] . \mathrm{Ca}^{2+}$ homeostasis undergoes subtle dysregulation in the physiologic aging. Disturbance of brain $\mathrm{Ca}^{2+}$ homeostasis may play a pivotal role in the causing of brain disease. Aging induces an increase in $\mathrm{Ca}^{2+}$ inflow by activating L-type voltage-sensitive $\mathrm{Ca}^{2+}$ channels and a decrease in $\mathrm{Ca}^{2+}$ efflux due to inhibiting the plasma membrane $\mathrm{Ca}^{2+}$-pumping activity ( $\mathrm{Ca}^{2+}$-ATPase) in brain neurons, leading to a derangement of $\mathrm{Ca}^{2+}$ homeostasis [16]. Increased intracellular $\mathrm{Ca}^{2+}$ may be transported to the mitochondria and endoplasmic reticulum. In addition, the increase in the brain microsomal $\mathrm{Ca}^{2+}$-sequestrating system $\left(\mathrm{Ca}^{2+}\right.$-ATPase $)$ with aging results in the microsomal calcium accumulation that is partly related to the brain toxicity by $\mathrm{Ca}^{2+}$. Intracellular $\mathrm{Ca}^{2+}$ signaling is fundamental to neuronal physiology and viability. Because of its ubiquitous roles, disruptions in $\mathrm{Ca}^{2+}$ homeostasis are implicated in diverse disease processes and have become a major focus of study in multifactorial neurodegenerative diseases such as Alzheimer's disease [17]. The mitochondrial $\mathrm{Ca}^{2+}-$ ATPase activity and its calcium content are not altered with aging. The endoplasmic reticulum (microsomes) $\mathrm{Ca}^{2+}$-ATPase activity is found to increase with aging, and the microsomal calcium accumulation is induced [18]. This enhancement may be related to $\mathrm{Ca}^{2+}$-dependent factors in the cytoplasm of neuronal cells. Protein kinase $\mathrm{C}$ activity in the cytoplasm is increased with aging, and this enzyme may activate the microsomal $\mathrm{Ca}^{2+}$-ATPase [18]. However, $\mathrm{Ca}^{2+}$-calmodulin dependent protein kinase may not be involved in aging-induced increase in the microsomal $\mathrm{Ca}^{2+}$ ATPase activity [18]. Regucalcin has been found to have an inhibitory effect on brain microsomal $\mathrm{Ca}^{2+}$-ATPase activity [10]. Brain microsomal $\mathrm{Ca}^{2+}$-ATPase activity is not significantly altered by calmodulin, calbindin or S-100A protein, which are $\mathrm{Ca}^{2+}$-binding proteins in brain [10]. The concentration of regucalcin in the brain tissues of young rats is about $5 \times 10^{-9} \mathrm{M}$ [19]. Brain microsomal $\mathrm{Ca}^{2+}$ ATPase activity was decreased with $10^{-9} \mathrm{M}$ which is a physiological concentration of regucalcin in the brain [10]. Meanwhile, regucalcin plays a role as an activator in the regulation of the mitochondrial $\mathrm{Ca}^{2+}$ -ATPase activity of rat brain tissues [20]. The addition of regucalcin $\left(10^{-10}\right.$ to $\left.10^{-8} \mathrm{M}\right)$ into the enzyme reaction mixture containing calcium chloride caused a significant increase in the mitochondrial $\mathrm{Ca}^{2+}$ ATPase activity [20]. Thus, regucalcin may play a physiologic role in the regulation of intracellular $\mathrm{Ca}^{2+}$ homeostasis in rat brain. The concentration of regucalcin in the cerebral cortex and hippocampus of brain tissues is decreased with aging [19]. Suppressive effect of regucalcin on brain microsomal $\mathrm{Ca}^{2+}$-ATPase activity has been found to weaken with aging [10]. Aging-induced elevation of brain microsomal $\mathrm{Ca}^{2+}$-ATPase activity may partly be resulted from an attenuation of the suppressive effect of regucalcin on the microsomal $\mathrm{Ca}^{2+}$-ATPase with aging, suggesting a pahophysiologic role of regucalcin in the regulation of brain intracellular $\mathrm{Ca}^{2+}$ homeostasis. Many functional proteins are degraded in the endoplasmic reticulum of brain neurons. Disturbance of $\mathrm{Ca}^{2+}$ homeostasis in the endoplasmic reticulum with aging may lead to an impairment of the degradation of modified proteins in brain neurons. The augmentation of modified proteins may promote cytotoxicity in the neurons.

$\mathrm{Ca}^{2+} /$ calmodulin-dependent protein kinases and protein kinase $\mathrm{C}$ play an important role in response of the cells to calcium signal in various cell types [21]. These protein kinases play a pivotal role in neuronal signaling processes [22]. Brain cytosolic $\mathrm{Ca}^{2+}$-dependent protein kinase activity is raised with increasing age [23]. Regucalcin has been shown to have an inhibitory effect on $\mathrm{Ca}^{2+} /$ calmodulin-dependent protein kinase and protein kinase $\mathrm{C}$ activities in the cytoplasm of brain tissues [23] and neurons [24]. Suppressive effects of regucalcin on brain $\mathrm{Ca}^{2+}$-dependent protein kinase activity may be weakened with increasing age [23]. Moreover, protein phosphatase plays an important role in intracellular signal transduction [25]. Regucalcin has been shown to have an inhibitory effect on $\mathrm{Ca}^{2+} /$ calmodulin-dependent protein phosphatase activity toward phosphotyrosine, phosphoserine

*Corresponding author: Masayoshi Yamaguchi, Department of Hematology and Medical Oncology, Emory University School of Medicine, 1365 C Clifton Road, NE Atlanta, GA 30322, USA, Tel: 404-664-7422; E-mail: yamamasa1155@yahoo.co.jp

Received October 14, 2013; Accepted October 15, 2013; Published October 21, 2013

Citation: Yamaguchi M (2013) Calcium Regulatory Protein, Regucalcin, may be a Key Molecule in Brain Disease. J Mol Genet Med 7: 87. doi: 10.4172/17470862.1000087

Copyright: () 2013 Yamaguchi M. This is an open-access article distributed under the terms of the Creative Commons Attribution License, which permits unrestricted use, distribution, and reproduction in any medium, provided the original author and source are credited 
and phosphothreonine in rat brain cytoplasm and neurons $[21,26]$. Calbindin and S-100A proteins, which are $\mathrm{Ca}^{2+}$-binding protein in brain [11], have no effect on protein phosphatase activity in rat brain cytoplasm [26]. Regucalcin is localized in the microsomes of rat brain, and aging causes a decrease in the microsomal regucalcin levels [27]. Brain microsomal protein tyrosine phosphatase activity is increased with increasing age [27]. This enzyme activity is found to be increased in the presence of anti-regucalcin monoclonal antibody in the enzyme reaction mixture, indicating that endogenous regucalcin in brain microsomes may have a suppressive effect on the enzyme activity [27]. The decrease in regucalcin in brain microsomes with aging is partly related to the enhancement of microsomal protein tyrosine phosphatase activity in the brain of aged rats. Interestingly, regucalcin has been found to be decreased in the nucleus of rat brain with aging [28]. Brain nuclear protein tyrosine phosphatase activity is increased in the presence of anti-regucalcin monoclonal antibody in the enzyme reaction mixture and this effect is deteriorated in aged rats [28]. These findings may be a result of the decrease in brain nuclear endogenous regucalcin levels with increasing age [28]. Thus, regucalcin plays a suppressive role in the regulation of protein tyrosine phosphatase activity in the nucleus of rat brain. Protein phosphatase may be important in the regulation of proteins phosphorylated by protein kinases [29]. Regucalcin may have a role in the control of dephosphorylation of phosphorylated proteins due to inhibiting protein tyrosine phosphatase in both the cytoplasm and nucleus of rat brain. Phosphorylated proteins may be important in the regulation of brain neuronal cell functions which are related to long-term potentiation with learning and memory [30]. The reduction of endogenous regucalcin in rat brain nucleus with aging may lead to the disturbance of brain functions.

Regucalcin has also been demonstrated to have a protective action against oxidative damage, without influencing antioxidant enzyme status in brain [31]. Moreover, regucalcin has been found to inhibit nitric oxide (NO) synthase activity in the cytosol of rat brain tissues [32]. NO synthase is activated by $\mathrm{Ca}^{2+} / \mathrm{calmodulin}$ which relates to $\mathrm{Ca}^{2+}$ signaling. $\mathrm{NO}$, which plays a role as a messenger or modulator molecule in various cell types, is produced by NO synthase [33]. Overproduction of NO may lead to the damage of various cells. Regucalcin may have a suppressive effect on overproduction of NO in the cells and may have a protective effect on NO-induced damage of cells. Regucalcin may regulate brain functions, which are related to NO, supporting the view that regucalcin plays a role as the regulatory protein in brain function. Disruptions in $\mathrm{Ca}^{2+}$ homeostasis are implicated in diverse disease processes and have become a major focus of study in neurodegenerative diseases such as Alzheiner disease [17]. A hall mark of Alzheimer disease is the excessive production of $\beta$-amyloid and its massive accumulation in amyloid plaques. Altered $\mathrm{Ca}^{2+}$ signaling accelerates amyloid-beta formation, whereas amyloidbeta peptides, particularly in soluble oligometric forms, induce $\mathrm{Ca}^{2+}$ disruptions [17]. A degenerative feed-forward cycle of toxic amyloidbeta generation and $\mathrm{Ca}^{2+}$ perturbations results, which in turn can spin off to accelerate more global neuropathological cascades, ultimately leading to synaptic breakdown, cell death, and devastating memory loss [17]. The amyloid hypothesis of Alzheimer's disease posits that the fundamental cause of Alzheimer's disease is the accumulation of the peptide amyloid-beta in the brain [34]. This hypothesis has been supported through observations that genetic defects in amyloid precursor protein and presenilin increase amyloid $\beta$ production and cause familial Alzheimer's disease [34].

Regucalcin has also been demonstrated to be involved in Parkinson's disease. Interestingly, the proteomic analysis ever of post-mortem locus ceruleus tissue of six pathologically confirmed Parkinson's disease patients, and six age- and gender-matched non-neurological controls showed that several individual proteins are identified that have hitherto not been associated with Parkinson's disease, such as regucalcin, which plays a role in maintaining intracellular $\mathrm{Ca}^{2+}$ homeostasis, and isoform 1 of kinectin, which is involved in transport of cellular components along microtubules [35]. In total 2495 proteins are identified, of which 87 proteins were differentially expressed in the locus ceruleus of Parkinson's disease patients compared with controls [35]. The majority of these differentially expressed proteins are known to be involved in processes that have been implicated in the pathogenesis of Parkinson's disease, including mitochondrial dysfunction, oxidative stress, protein misfolding, cytoskeleton dysregulation, and inflammation [14]. Moreover, the neurofibrillary tangles (NFTs) formed by the accumulation of abnormal tau filaments have been shown to be involved in Alzheimer's disease brain degeneration. A tau transgenic mouse (pNSE/htau23) model was used to monitor changes in protein levels and to search for novel biomarker candidates suitable for the early diagnosis of Alzheimer's disease before onset of clinical symptoms [36]. Plasma samples from 2-month (asymptomatic) and 4-month (symptomatic) tau transgenic mice were compared to the control group by 2 -dimensional gel electrophoresis coupled with liquid chromatography-tandem mass spectrometry. Three proteins, ATP synthase, adenosine kinase and regucalcin showed significantly decreased levels in the plasma of tau transgenic mouse, which was further confirmed by Western blotting [36]. This study suggests that regucalcin could be used as candidate biomarkers for early diagnosis of Alzheimer's disease in combination with previously discovered protein biomarkers. Thus, regucalcin may play an important role in revelation of brain disease including Alzheimer's and Parkinson's disease.

As described above, regucalcin has been shown to play a multifunctional role as a regulatory protein in cell signaling in brain neuronal cells $[6,7]$. In this aspect, regucalcin has been demonstrated to play in the regulation of $\mathrm{Ca}^{2+}$ homeostasis and $\mathrm{Ca}^{2+}$ signaling in brain neurons; the protein reveals suppressive effects on microsomal $\mathrm{Ca}^{2+}$ pump activity, $\mathrm{Ca}^{2+}$-dependent protein kinases, protein phosphatases and NO synthase activities in the brain of rats. Brain regucalcin concentration is decreased with aging, and the regulatory effects of regucalcin in brain neuronal cell functions are weakened with increasing age. The decrease in regucalcin gene expression with aging may be implicated to leading to neurodegenerative diseases such as Alzheimer's and Parkinson's diseases. Regucalcin may be a target molecule in brain diseases with aging. Development of further studies will be expected.

\section{Author Contribution and Disclosures}

\section{The author has no conflicts of interest.}

\section{Acknowledgement}

Regucalcin studies of the author was supported by a Grant-in-Aid for Scientific Research (C) No.63571053, No.02671006, No.04671362, No.06672193 No.08672522, No.10672048, No.13672292 and No.17590063 from the Ministry of Education, Science, Sports, and Culture, Japan. Also, the author was awarded from the Bounty of Encouragement Foundation in Pharmaceutical Research and the Bounty of the Yamanouchi Foundation for Research on Metabolic Disorders. This study was also supported by the Foundation for Biomedical Research on Regucalcin.

\section{References}

1. Yamaguchi M, Yamamoto $T$ (1978) Purification of calcium binding substance from soluble fraction of normal rat liver. Chem Pharm Bull (Tokyo) 26: 19151918. 
Citation: Yamaguchi M (2013) Calcium Regulatory Protein, Regucalcin, may be a Key Molecule in Brain Disease. J Mol Genet Med 7: 87. doi: 10.4172/1747-0862.1000087

2. Yamaguchi M, Mori S (1988) Effect of $\mathrm{Ca}^{2+}$ and $\mathrm{Zn}^{2+}$ on 5'-nucleotidase activity in rat liver plasma membranes: Hepatic calcium-binding protein (regucalcin) reverses the $\mathrm{Ca}^{2+}$ effect. Chem Pharm Bull 36: 321-325.

3. Yamaguchi M (1992) A novel $\mathrm{Ca}^{2+}$-binding protein regucalcin and calcium inhibition. Regulatory role in liver cell function. In: Calcium Inhibition. Japan Sci Soc Press, Tokyo and CRC Press, Boca Raton pp19-41.

4. Shimokawa N, Yamaguchi M (1993) Molecular cloning and sequencing of the cDNA coding for a calcium-binding protein regucalcin from rat liver. FEBS Let 327: 251-255.

5. Yamaguchi M (2011) The transcriptional regulation of regucalcin gene expression. Mol Cell Biochem 346: 147-171.

6. Yamaguchi M (2005) Role of regucalcin in maintaining cell homeostasis and function (review). Int J Mol Med 15: 371-389.

7. Yamaguchi M (2011) Regucalcin and cell regulation: role as a suppressor protein in signal transduction. Mol Cell Biochem 353: 101-137.

8. Shimokawa N, Matsuda Y, Yamaguchi M (1995) Genomic cloning and chromosomal assignment of rat regucalcin gene. Mol Cell Biochem 151: 157-163.

9. Thiselton DL, McDowall J, Brandau O, Ramser J, d'Esposito F, et al. (2002) An integrated, functionally annotated gene map of the DXS8026-ELK1 interval on human Xp11.3-Xp11.23: potential hotspot for neurogenetic disorders. Genomics 79: 560-572

10. Yamaguchi M, Hanahisa Y, Murata T (1999) Expression of calcium-binding protein regucalcin and microsomal $\mathrm{Ca}^{2+}$-ATPase regulation in rat brain: attenuation with increasing age. Mol Cell Biochem 200: 43-49.

11. Yamaguchi $M(2012)$ Role of regucalcin in brain calcium signaling: involvement in aging. Integr Biol (Camb) 4: 825-837.

12. Wojda U, Salinska E, Kuznicki J (2008) Calcium ions in neuronal degeneration IUBMB Life 60: 575-590.

13. Heizmann CW, Braun $\mathrm{K}$ (1992) Changes in $\mathrm{Ca}^{2+}$-binding proteins in human neurodegenerative disorders. Trends Neurosci 15: 259-264.

14. Dahan D, Spanier R, Rahamimoff $H$ (1991) The modulation of rat brain $\mathrm{Na}^{+}$ $\mathrm{Ca}^{2+}$ exchange by $\mathrm{K}^{+}$. J Biol Chem 266: 2067-2075.

15. Cambray-Deakin MA, Burgoyne RD (1992) Intracellular $\mathrm{Ca}^{2+}$ and $\mathrm{N}$-methylD-aspartate-stimulated neuritogenesis in rat cerebellar granule cell cultures. Brain Res Dev Brain Res 66: 25-32.

16. Norris CM, Blalock EM, Chen KC, Porter NM, Landfield PW (2002) Calcineurin enhances L-type $\mathrm{Ca}^{2+}$ channel activity in hippocampal neurons: increased effect with age in culture. Neuroscience 110: 213-225.

17. Demuro A, Parker I, Stutzmann GE (2010) Calcium signaling and amyloid toxicity in Alzheimer disease. J Biol Chem 285: 12463-12468.

18. Hanahisa $Y$, Yamaguchi M (1998) Increase of $\mathrm{Ca}^{2+}$-ATPase activity in the brain microsomes of rats with increasing ages: involvement of protein kinase $\mathrm{C}$. Brain Res Bull 46: 329-332.

19. Yamaguchi M, Isogai M (1993) Tissue concentration of calcium-binding protein regucalcin in rats by enzyme-linked immunoadsorbent assay. Mol Cell Biochem 122: $65-68$.

20. Yamaguchi M, Takakura Y, Nakagawa T (2008) Regucalcin increases $\mathrm{Ca}^{2+}$ ATPase activity in the mitochondria of brain tissues of normal and transgenic rats. J Cell Biochem 104: 795-804.

21. Hamano T, Yamaguchi M (1999) Inhibitory effect of regucalcin on $\mathrm{Ca}^{2+}$ calmodulin-dependent protein phosphatase activity in rat brain cytosol. Int $\mathrm{J}$ Mol Med 3: 615-619.

22. Abdul HM, Sama MA, Furman JL, Mathis DM, Beckett TL, et al. (2009) Cognitive decline in Alzheimer's disease is associated with selective changes in calcineurin/NFAT signaling. J Neurosci 29: 12957-12969.

23. Hamano T, Hanahisa Y, Yamaguchi M (1999) Inhibitory effect of regucalcin on $\mathrm{Ca}^{2+}$-dependent protein kinase activity in rat brain cytosol: involvement of endogenous regucalcin. Brain Res Bull 50: 187-192.
24. Hamano T, Yamaguchi M (2001) Inhibitory role of regucalcin in the regulation of $\mathrm{Ca}^{2+}$ dependent protein kinases activity in rat brain neurons. J Neurol Sci 183: 33-38.

25. Wang Y, Santini F, Qin K, Huang CY (1995) A Mg ${ }^{2+}$-dependent, $\mathrm{Ca}^{2+}$-inhibitable serine/threonine protein phosphatase from bovine brain. J Biol Chem 270 : 25607-25612.

26. Yamaguchi M, Hamano T, Misawa $\mathrm{H}$ (2000) Expression of $\mathrm{Ca}^{2+}$-binding protein regucalcin in rat brain neurons: inhibitory effect on protein phosphatase activity. Brain Res Bull 52: 343-348.

27. Tobisawa M, Tsurusaki Y, Yamaguchi M (2003) Decrease in regucalcin level and enhancement of protein tyrosine phosphatase activity in rat brain microsomes with increasing age. Int J Mol Med 12: 577-580.

28. Tobisawa M, Yamaguchi M (2003) Suppressive effect of endogenous regucalcin on protein tyrosine phosphatase activity in the nucleus of rat brain: attenuation with increasing age. Int J Mol Med 11: 205-210.

29. Loh C, Shaw KT, Carew J, Viola JP, Luo C, et al. (1996) Calcineurin binds the transcription factor NFAT1 and reversibly regulates its activity. J Biol Chem 271: 10884-10891.

30. De Lorenzo RJ, Freedman SD, Yoke WB, Maurer SC (1979) Stimulation of $\mathrm{Ca}^{2+}$-dependent neurotransmitter release and presynaptic nerve terminal protein phosphorylation by calmodulin and a calmodulin-like protein isolated synaptic vesicle. Proc Nstl Acad Sci USA 76: 1838-1842.

31. Son TG, Zou Y, Jung KJ, Yu BP, Ishigami A, et al. (2006) SMP30 deficiency causes increased oxidative stress in brain. Mech Ageing Dev 127: 451-457.

32. Tobisawa M, Yamaguchi M (2003) Inhibitory role of regucalcin in the regulation of nitric oxide synthase activity in rat brain cytosol: involvement of aging. $J$ Neurol Sci 209: 47-54.

33. Tobisawa M, Yamaguchi M (2003) Role of endogenous regucalcin in brain function: Suppression of cytosolic nitric oxide synthase and nuclear protein tyrosine phosphatase activities in brain tissue of transgenic rats. Int J Mol Med 12: $581-585$

34. Bartzokis G (2011) Alzheimer's disease as homeostatic responses to agerelated myelin breakdown. Nerubiol Aging 32: 1341-1371.

35. van Dijk KD, Berendse HW, Drukarch B, Fratantoni SA, Pham TV, et al. (2012) The proteome of the locus ceruleus in Parkinson's disease: relevance to pathogenesis. Brain Pathol 22: 485-498.

36. Kim YH, Lee EK, Park SA, Kim NH, Kim CW (2012) Proteomic analysis of plasma from a Tau transgenic mouse. Int J Dev Neurosci 30: 277-283. 